\title{
Real-Time Pricing for Demand Response in Smart Grid Based on Alternating Direction Method of Multipliers
}

\author{
Hongbo Zhu, ${ }^{1,2}$ Yan Gao $\mathbb{D}^{1},{ }^{1}$ and Yong $\mathrm{Hou}^{3,4}$ \\ ${ }^{1}$ School of Management, University of Shanghai for Science and Technology, Shanghai 200093, China \\ ${ }^{2}$ Faculty of Mathematics and Physics, Huaiyin Institute of Technology, Huai'an 223003, China \\ ${ }^{3}$ Institute for Energy Studies, University of North Dakota, Grand Forks, ND 58202, USA \\ ${ }^{4}$ Clean Republic LLC, Grand Forks, ND 58302, USA \\ Correspondence should be addressed to Yan Gao; gaoyan@usst.edu.cn
}

Received 10 May 2017; Revised 13 December 2017; Accepted 31 December 2017; Published 29 January 2018

Academic Editor: Bogdan Dumitrescu

Copyright (c) 2018 Hongbo Zhu et al. This is an open access article distributed under the Creative Commons Attribution License, which permits unrestricted use, distribution, and reproduction in any medium, provided the original work is properly cited.

\begin{abstract}
The real-time pricing (RTP) scheme is an ideal method to adjust the power balance between supply and demand in smart grid systems. This scheme has a profound impact on users' behavior, system operation, and overall grid management in the electricity industry. In this research, we conduct an extended discussion of a RTP optimization model and give a theoretical analysis of the existence and uniqueness of the Lagrangian multiplier. A distributed optimization method based on the alternating direction method of multipliers (ADMM) algorithm with Gaussian back substitution (GBS) is proposed in this study. On the one hand, the proposed algorithm takes abundant advantage of the separability among variables in the model. On the other hand, the proposed algorithm can not only speed up the convergence rate to enhance the efficiency of computing, but also overcome the deficiency of the distributed dual subgradient algorithm, the possibility of nonconvergence in the iteration process. In addition, we give the theoretical proof of the convergence of the proposed algorithm. Furthermore, the interdependent relationship between variables has been discussed in depth during numerical simulations in the study. Compared with the dual subgradient method, the simulation results validate that the proposed algorithm has a higher convergence speed and better implementation effect.
\end{abstract}

\section{Introduction}

1.1. Background and Motivation. In 2007, the United States Congress passed the Energy Independence and Security Act. It claims that the specific goals for the development of the nation's smart grid are to conserve energy and reduce emission. On the one hand, it promotes the development of alternative energy sources, such as hydro, biomass, and wind. The rapid development of renewable energy has brought about wide applications in the power generation industry. On the other hand, to bring about reduction in carbon emission, the use of smart appliances as plug-in vehicles and hot pumps is encouraged. So far, the capability of supplying electric power in developed and most of the developing countries can meet the normal demand load of consumers, except in the peak load periods. Even if an outage happens a few times per year in peak load periods, it will potentially cause huge damage in our economic and social system, along with an unacceptable cost to recover from this damage. For example, the well-known "8-14" blackout in USA and Canada brought about a dramatic economic loss for both countries in 2003. To mitigate outages, nations must invest huge capital to meet the peak demand. For example, in the United States, the peak demand sums up to only 100 hours per year, but it accounts for $10-20 \%$ of the electricity cost in the whole electric power industry annually. In the National Electricity Market of Australia, $20-30 \%$ of the electricity network capacity or $\$ 60$ billion is spent on meeting, no more than 90 hours, the peak demand in a year [1]. This indicates that the most important cost for electricity is invested by how to meet the peak power demand. The most effective way to solve this problem is to cut the peak and fill the valley, which is also the objective that research on demand side management (DSM) in smart grid should achieve.

DSM mechanism based on price can effectively motivate electricity consumers to adjust their inherent mode of using 
electricity and to change their behaviors with the smart grid system through following varying price signals of electricity [2]. For example, an individual customer may change his/her air conditioner temperature or reprogram the charging process of his/her electric car or/and battery banks during the valley hours, while his/her watt hour bill of electricity is the lowest. These types of changes may help us achieve the objective of cutting peak load in demand side. Customers are motivated to participate in DSM through the implementation of appropriate pricing schemes such as peak load pricing, time-of-use (TOU) pricing, and real-time pricing (RTP). For instance, a joint optimization model of generation side and user side based on TOU pricing in China has been discussed by Tan et al. [3]. But RTP has been accepted as the most effective pricing scheme in DSM [4].

The RTP principle was initially proposed by Caramanis et al. in the 1980s [5]. It has widely been recognized as one of the promising solutions more effective in making both customers and suppliers satisfied during peak reduction. Different from other existing pricing schemes, by RTP schemes, the price is determined prior to the deal happening. The RTP system, through intercommunication between the supply and demand of electricity, can realize peak shaving.

1.2. Related Work. Aiming at maximizing aggregated welfare for the users as well as minimizing cost of the providers, Samadi et al. adopted the pricing scheme and proposed multiple innovative RTP models [6-8]. These models have been paid much attention and cited by a lot of researchers in the past several years. Based on Mohsenian-Rad's models, many extended models have been proposed with respect to different situations. Song and $\mathrm{Qu}$ altered the optimization model of Mohsenian-Rad, through substituting the quadratic utility function with a logarithmic utility function [9]. Tarasak added a load uncertainty factor into RTP models and, with consideration of three different uncertainties, classified them into bounded uncertainty model, Gaussian model, and unknown distribution model [10]. Wang et al. improved the RTP model through subtracting a power fluctuation factor in the objective function and then analyzed the system from both offline and online aspects [11]. Asadi et al. considered a system with one single supplier and multiple users, which includes several commercial and residential subscribers [12].

A good smart grid system requests information exchange between suppliers and consumers at a super high efficient level. A fundamental challenge remains for researchers in the field: How can a model be developed to meet the demand of exchanging information in a smart grid system and at the same time not sacrifice the private information of the individual users? In order to meet the challenge, a distributed dual subgradient algorithm has been widely applied in mathematic models by researchers [6-12]. The distributed dual subgradient algorithm simulates the dynamic process between users and energy providers, and at the end its dynamic process converges to an equilibrium point, which obtains an optimal solution that gives the best choice to buyers and sellers in the whole energy business deal. By exploring the distributed dual subgradient algorithm, a lot of good results have been achieved in the research of
RTP models. However, the algorithm still presents some problems that are worth further discussion. For example, it suffers in its optimizing process with slow convergence, high sensitivity to the step size, or/and even nonconvergence sometimes [1]. To overcome the deficiencies in the subgradient algorithm, Asadi et al. employed the particle swarm optimization algorithm in their RTP model [12]. Zhang et al. proposed a fast-distributed dual gradient algorithm [1]. Furthermore, a lot of research exploited the alternating direction method of multipliers (ADMM) algorithm and declared that this algorithm can not only guarantee the distributed advantage of the dual subgradient algorithm, but also avoid the shortcomings of slow convergence or even nonconvergence [13-17]. Tsai et al. proposed a randomized ADMM approach to achieve real-time power balance in a neighborhood with a large number of load customers [13]. Tan et al. discussed three distributed ADMM algorithms for solving the dynamic DC optimal power flow problem with demand response [14]. A short-term control strategy for active power distribution systems was proposed to regulate voltages into statutory ranges [15]. Wang et al. proposed a consensus-based ADMM method for solving the dynamic DC optimal power flow problem with demand response in a distributed manner [16]. The total welfare in the grid was maximized and the optimization problem was analytically solved using the ADMM and consensus theory for multiagent systems [17].

However, some researches do not take into account the fact that direct extension of ADMM method to an optimization model with more than two variables cannot guarantee its convergence, such as researches conducted by Tsai et al. [13] and by Tan et al. [14]. The models developed in $[15,16]$ solved the problems of demand response well, but they do not involve the RTP scheme. Noticing that the dual subgradient algorithm used in [6-12] was a gradientbased method which usually had a slow convergence time, [17] developed a distributed approach based on ADMM. Comparing the convergence of the two algorithms, the superiority of the designed algorithm will be reflected more.

Aiming at solving the maximization problem of the social welfare model and avoiding nonconvergence of the dual subgradient algorithm, we proposed a distributed optimization algorithm based on ADMM with Gaussian back substitution (ADMM-GBS). The ADMM-GBS algorithm can effectively guarantee that the convergence is reached and ensure that the independent distribution in the user's side is achieved. The main novelty of this research can be summarized as follows.

(i) An extended discussion of a RTP optimization model is developed to give theoretical analysis of the existence and uniqueness of the Lagrangian multiplier.

(ii) From the global consideration, fully utilizing the separability among variables, a distributed optimization algorithm based on ADMM-GBS to solve the RTP model is proposed. This algorithm can not only speed up the convergence rate to enhance the efficiency of computing, but also overcome widely known deficiencies in the possibility of nonconvergence in the iteration process. In addition, the convergence of the algorithm based on ADMM-GBS is given by the study. 
(iii) The interdependent relationship between variables is discussed in depth during numerical simulations in the study. Compared with the dual subgradient method, the simulation results validate that the proposed algorithm has a faster convergence speed and better implementation effect.

The remainder of the paper is organized as follows. The system model and optimization problem are presented in Section 2. In Section 3, the research discusses the extension of the RTP optimization model in detail by attaching a Lagrangian multiplier to the problem, and furthermore it clarifies the existence and the uniqueness of the Lagrangian multiplier in the model. The distributed optimization algorithm based on ADMM-GBS is proposed in Section 4; in addition, the convergence of the proposed algorithm is given. In Section 5, the performance of the ADMM-GBS algorithm is simulated and validated, along with a deep discussion of the interdependent relationship between model variables. In Section 6, this study reaches a conclusion with respect to the effectiveness and feasibility of using the proposed algorithm to solve the RTP model.

\section{System Model}

Normally, we consider an electric power distribution system that consists of a single energy provider and several load subscribers or users. An energy provider and all users are connected with each other through an information communication infrastructure. Following notations broadly accepted by literatures, we define the notation system of this research below. A whole cycle is divided into $K$ time slots. For each user $i \in \mathbb{N}=\{1,2, \ldots, N\}, k \in \mathbb{K}=\{1,2, \ldots, K\}$, let $x_{i}(k)$ be the power consumption demand at time slot $k$ of customer $i$. The range of $x_{i}(k)$ is $\left[m_{i}^{k}, M_{i}^{k}\right]$, where $m_{i}^{k} \geq 0$ and $M_{i}^{k} \geq 0$ correspond to the minimum and the maximum consumption in time slot $k$ of customer $i$, respectively.

2.1. Utility Function of Users. Each user is assumed to behave independently in a power system. They have their own preferences for energy demand and time schedules upon various prices. The concept of utility function $U\left(x_{i}(k)\right)$ derived from microeconomics is employed to reflect the level of satisfaction obtained by user $i$ in consuming $x_{i}(k)$ amount of energy at time slot $k$.

We assume that the utility function is nondecreasing and concave. Generally, this study exploits the following utility function which derives from multiple researches and is being widely adopted $[6-8,10-12]$ :

$$
\begin{aligned}
& U_{i}\left(x_{i}(k)\right) \\
& = \begin{cases}\omega_{i}(k) x_{i}(k)-\frac{\alpha_{i}}{2} x_{i}(k)^{2}, & \text { if } 0 \leq x_{i}(k) \leq \frac{\omega_{i}(k)}{\alpha_{i}}, \\
\frac{\omega_{i}(k)^{2}}{2 \alpha_{i}}, & \text { if } x_{i}(k) \geq \frac{\omega_{i}(k)}{\alpha_{i}},\end{cases}
\end{aligned}
$$

where $i \in \mathbb{N}$ and $\alpha_{i}>0$ is a predetermined parameter. Herein, the parameter $\omega_{i}(k)>0$ indicates the preference of user $i$ at time slot $k$ and may vary at different time slots. A larger $\omega_{i}(k)$ means a higher level of flexibility. The utility function is relative to private information of users, which can possibly be utilized to reconstruct the aspects of users' consumption behavior daily [18].

2.2. Cost Function of the Energy Provider. Generally, based on the widely accepted modeling description and the power system this research is observing, we consider the following quadratic function as the cost model of the energy provider [6-12]:

$$
C_{k}\left(G_{k}\right)=a_{k} G_{k}^{2}+b_{k} G_{k}+c_{k}
$$

where $G_{k} \in \mathbb{G}=\left[G_{k}^{\min }, G_{k}^{\max }\right], \forall k \in \mathbb{K}, G_{k}^{\min }=\sum_{i=1}^{N} m_{i}^{k}$, and $G_{k}^{\max }=\sum_{i=1}^{N} M_{i}^{k}, a_{k}>0$, and $b_{k}, c_{k} \geq 0$ are predetermined constants.

2.3. Optimization Model. The interactions between the energy provider and its customers can be formulated as optimization problems in various models based on different scenarios. A RTP model was proposed by Samadi et al. as maximizing the aggregated welfare of users as well as minimizing the cost of the provider [6]. Mathematically, for each time slot $\forall k \in \mathbb{K}$, the optimization model of RTP can be expressed as

$$
\begin{array}{ll}
\max & \sum_{i=1}^{N} U_{i}\left(x_{i}(k)\right)-C_{k}\left(G_{k}\right) \\
\text { s.t. } & \sum_{i=1}^{N} x_{i}(k) \leq G_{k}, \\
& m_{i}^{k} \leq x_{i}(k) \leq M_{i}^{k}, \\
& G_{k}^{\min } \leq G_{k} \leq G_{k}^{\max } .
\end{array}
$$

The first constraint ensures that, at time slot $k$, the total actual power consumption cannot exceed the energy provided by the energy supplier. The other two constraints bound the range of variables $x_{i}(k)$ and $G_{k}$. Since the function $U_{i}(\cdot)$ is concave, $C_{k}(\cdot)$ is convex and the constraints are defined by affine functions. It can be verified that problem (3) is a convex optimization problem.

In order to avoid using the centralized algorithm and to take full advantage of the infrastructure of information and communication technology in smart grids, a distributed optimization algorithm based on ADMM-GBS will be presented in Section 4. In fact, prior to employing the ADMM algorithm, a reformulation of problem (3) to a suitable form should be made first [19].

In the actual circumstance, the demand on electricity of customers directed by the varying price is not saturated. It is reasonable to believe that due to the characteristic of monotonous increment of utility function, more consumption of electricity, and more social welfare brought about to households inevitably. Therefore, we can conclude that the solution will be reached at the boundary. This means the constraint condition can be simplified such that the aggregated load of users at time slot $k$ should be equal to the energy provided by the supplier at the same time slot. 
Then, the RTP maximization model (3) is equivalent to (4) as follows:

$$
\begin{array}{ll}
\min & C_{k}\left(G_{k}\right)-\sum_{i=1}^{N} U_{i}\left(x_{i}(k)\right) \\
\text { s.t. } & \sum_{i=1}^{N} x_{i}(k)=G_{k}, \\
& m_{i}^{k} \leq x_{i}(k) \leq M_{i}^{k}, \\
& G_{k}^{\min } \leq G_{k} \leq G_{k}^{\max } .
\end{array}
$$

In the equality-constrained optimization problem (4), the cost function with respect to $G_{k}$ is convex; the utility function with respect to $x_{i}(k)$ is concave. This reformulated formulation fits into the ADMM framework depicted by Boyd et al. [19]. Hence, in the following, this study will develop a distributed optimization algorithm based on ADMM-GBS to solve problem (4).

\section{Preliminary Knowledge}

3.1. General Form of ADMM. The ADMM can effectively decompose the minimization problem into smaller subproblems and then coordinate solutions from subproblems. Through this decomposition-coordination process, we may end up obtaining a global solution of the original problem. This algorithm was firstly proposed in the late 1960s by Hestenes [20] and Powell [21] and recently reintroduced by Boyd et al. [19]. The general form of ADMM is presented for the following problem:

$$
\begin{aligned}
& \min \quad f(x)+g(z) \\
& \text { s.t. } \quad A x+B z=c,
\end{aligned}
$$

with variables $x \in R^{n}$ and $z \in R^{m}$, where $A \in R^{p \times n}, B \in R^{p \times m}$, and $c \in R^{p}$. Assume that $f$ and $g$ are convex functions.

The augmented Lagrangian function associated with problem (5) is

$$
\begin{aligned}
L_{\rho}(x, z, \lambda)= & f(x)+g(z)-\lambda^{T}(A x+B z-c) \\
& +\frac{\rho}{2}\|A x+B z-c\|^{2},
\end{aligned}
$$

where $\lambda \in R^{m}$ is the Lagrangian multiplier and $\rho>0$ is the parameter for the quadratic penalty item of the constraint. This penalty item guarantees convergence of the objective function without requiring strict assumption conditions like strict convexity or finiteness. The iterative scheme of ADMM for (5) embeds a Gauss-Seidel decomposition into iterations of $x$ and $z$ as follows:

$$
\begin{aligned}
& x^{t+1}=\arg \min _{x} L_{\rho}\left(x, z^{t}, \lambda^{t}\right), \\
& z^{t+1}=\arg \min _{z} L_{\rho}\left(x^{t+1}, z, \lambda^{t}\right), \\
& \lambda^{t+1}=\lambda^{t}+\rho\left(A x^{t+1}+B z^{t+1}-c\right) .
\end{aligned}
$$

The augmented Lagrangian is minimized over $x$ and $z$ separately during each process of the iteration. In (7), functions $f$ and $g$ as well as variables $x$ and $z$ are treated individually, so the subproblems can be easily generated. This feature is quite attractive and advantageous for solving the RTP optimization problem. The convergence of ADMM for the convex optimization problem with such two blocks of variables and functions has been given by Boyd et al. [19].

3.2. Lagrangian Multiplier. In order to bring about robustness to the subgradient algorithm and to yield convergence without assumptions like strict convexity or finiteness of the objective function, the augmented Lagrangian function has been employed by the research. To decompose the problem, by attaching a Lagrange multiplier $\lambda$ into a linear constraint, the augmented Lagrangian function for problem (4) can be given by

$$
\begin{aligned}
L_{\rho}\left(X_{k}, G_{k}, \lambda_{k}\right)= & C_{k}\left(G_{k}\right)-\sum_{i=1}^{N} U_{i}\left(x_{i}(k)\right) \\
& -\lambda_{k}\left(G_{k}-\sum_{i=1}^{N} x_{i}(k)\right) \\
& +\frac{\rho}{2}\left\|G_{k}-\sum_{i=1}^{N} x_{i}(k)\right\|^{2},
\end{aligned}
$$

where $X_{k}=\left(x_{1}(k), x_{2}(k), \ldots, x_{N}(k)\right)$ and $\lambda_{k} \geq 0$ (the Lagrangian multiplier $\lambda_{k}$ associated with the inequality constraint problem (3) is nonnegative; the uniqueness of the multiplier will be clarified subsequently in Remark 1; the uniqueness guarantees that the multiplier of equality constraint problems (4) that satisfies the condition $\lambda_{k} \geq 0$ for (4) has the same solution as (3)) is the Lagrangian multiplier to embody the shadow price in economics and is often used to indicate the electricity price. Note that

$$
\begin{aligned}
& L_{0}\left(X_{k}, G_{k}, \lambda_{k}\right) \\
& \quad=C_{k}\left(G_{k}\right)-\sum_{i=1}^{N} U_{i}\left(x_{i}(k)\right)-\lambda_{k}\left(G_{k}-\sum_{i=1}^{N} x_{i}(k)\right) .
\end{aligned}
$$

Formula (9) is the standard, nonaugmented Lagrangian function associated with (4). The augmented Lagrangian (8) can be depicted as (9) associated with the following problem:

$$
\begin{array}{ll}
\min & C_{k}\left(G_{k}\right)-\sum_{i=1}^{N} U_{i}\left(x_{i}(k)\right)+\frac{\rho}{2}\left\|G_{k}-\sum_{i=1}^{N} x_{i}(k)\right\|^{2} \\
\text { s.t. } & \sum_{i=1}^{N} x_{i}(k)=G_{k} .
\end{array}
$$

When the penalty term added to the objective function is zero for any feasible $x_{i}(k)$, problem (10) is clearly equivalent to the original problem (4). In this specific problem, the penalty item depicts the uncertainty of the use of electricity. The benefit of involving a penalty term in the minimization 
problem is that the corresponding function (10) can be verified to be differentiable under rather mild conditions on the original problem.

Next, we give the detailed analysis of the existence and uniqueness of the Lagrangian multiplier associated with the inequality constraints problem (3).

\section{Remark 1 .}

(i) Existence. The optimization problem of RTP (3) has been modeled on a practical problem in the system of smart grid. Therefore, there exists an optimal solution. This conclusion has been illustrated by Samadi et al. [6]. Deriving from the saddle point theorem, the existence of an optimal solution is the necessary and sufficient condition of existence of a saddle point [22]. Thus, we should reach a conclusion that a Lagrangian multiplier satisfying Slater's condition is existent for convex optimization problem (3).

(ii) Uniqueness. Suppose that, for given $\bar{G}_{k} \in \mathbb{G} \subseteq$ $R$, the local optimal solution of problem (3) is $\bar{X}_{k}=$ $\left(\bar{x}_{1}(k), \bar{x}_{2}(k), \ldots, \bar{x}_{N}(k)\right) \in R^{N}$ and the related Lagrangian multiplier is $\bar{\lambda}_{k} \geq 0$. Obviously, $\left(\bar{x}_{i}(k), \bar{G}_{k}, \bar{\lambda}_{k}\right)$ is the saddle point, and it satisfies the KKT conditions with multiplier $\lambda_{k}$ depicted as follows:

$$
\begin{aligned}
U_{i}^{\prime}\left(\bar{x}_{i}(k)\right)-\bar{\lambda}_{k} & =0, \quad i \in \mathbb{N}, \\
C_{k}^{\prime}\left(\bar{G}_{k}\right)-\bar{\lambda}_{k} & =0, \\
\bar{\lambda}_{k}\left(\bar{G}_{k}-\sum_{i=1}^{N} \bar{x}_{i}(k)\right) & =0, \\
\bar{G}_{k}-\sum_{i=1}^{N} \bar{x}_{i}(k) & \geq 0, \\
\bar{\lambda}_{k} & \geq 0 .
\end{aligned}
$$

Given $g\left(X_{k}, G_{k}\right)=\sum_{i=1}^{N} x_{i}(k)-G_{k}$, we prove that the following three conditions are satisfied.

(a) Second-Order Sufficient Condition. For $\nabla_{X_{k}}^{2} L_{0}\left(\bar{X}_{k}, \bar{G}_{k}, \bar{\lambda}_{k}\right)$ $=\operatorname{diag}\left(\alpha_{1}, \alpha_{2}, \ldots, \alpha_{N}\right), \alpha_{i}>0$, we conclude that $\nabla_{X_{k}}^{2} L_{0}\left(\bar{X}_{k}\right.$, $\left.\bar{G}_{k}, \bar{\lambda}_{k}\right)$ is a positive definite matrix. Inevitably, $\left\langle y, \nabla_{X_{k}}^{2} L_{0}\left(\bar{X}_{k}\right.\right.$, $\left.\left.\bar{G}_{k}, \bar{\lambda}_{k}\right) y\right\rangle>0, y \neq 0, y \in C_{k}^{\bar{S}}\left(\bar{X}_{k}, \bar{G}_{k}\right)$, is satisfied, where

$$
\begin{aligned}
& C_{k}^{\bar{S}}\left(\bar{X}_{k}, \bar{G}_{k}\right)=\left\{y \in R^{n} \mid\left\langle\nabla_{X} g\left(\bar{X}_{k}, \bar{G}_{k}\right), y\right\rangle=0, \bar{\lambda}_{k}\right. \\
& \quad>0,\left\langle\nabla_{X_{k}} g\left(\bar{X}_{k}, \bar{G}_{k}\right), y\right\rangle \leq 0, g\left(\bar{X}_{k}, \bar{G}_{k}\right)=0, \bar{\lambda}_{k} \\
& \quad \leq 0\} .
\end{aligned}
$$

(b) Linearly Independent Constraint Specification. There is only one constraint condition, and the gradient of the constraint condition is not equal to zero. This means $\nabla_{X_{k}} g\left(\bar{X}_{k}, \bar{G}_{k}\right) \neq 0$, and the constraint condition is linearly independent. (c) Strict Complementarity. It is obvious, based on the definition of shadow price and the model established on a practical problem of smart grid, that the condition is satisfied. With the increment of electricity consumption which produces more social welfare, inevitably, the utility function increases monotonously. This means that $g\left(\bar{X}_{k}, \bar{G}_{k}\right)=0$ is equivalent to $\bar{\lambda}_{k}>0$.

Derived by Theorem 3.32 [23], we can conclude that, in the neighborhood $\Omega \in \mathbb{G}$ of $\bar{G}_{k}$, there exist continuous differentiable functions $x_{i}(\cdot): \Omega \rightarrow R$ and $\lambda_{k}(\cdot): \Omega \rightarrow R$ satisfying conditions $x_{i}\left(\bar{G}_{k}\right)=\bar{x}_{i}(k)$ and $\lambda_{k}\left(\bar{G}_{k}\right)=\bar{\lambda}_{k}$, respectively. It is presented that both the local optimal solution and the related Lagrangian multiplier exist uniquely in a local region, while the parameter variable $\bar{G}_{k}$ varies a little. In summary, the description of this remark is established.

Remark 1 clarifies that the optimization problem (3) has a unique nonnegative Lagrangian multiplier. It ensures that the equality-constrained optimization problem (4) has a unique nonnegative multiplier. The solution of (3) can be obtained through solving (4) due to the equivalence with two problems.

\section{Distributed Optimization Algorithm Based on ADMM-GBS}

The ADMM is an effective tool for solving the convex optimization problem of two separable variables. It has been well studied in previous research [20-24]. However, directly extending ADMM method into an optimization model with more than two variables cannot guarantee its convergence. It is necessary to amend it. Setzer et al. and He and Yuan amended the basic ADMM model and analyzed some interesting applications in more general cases where its variables are more than two $[25,26]$.

In light of the improved methods [26], we propose a distributed optimization algorithm based on ADMM-GBS to solve the RTP problem (4). We first give the definitions of several matrices which will be used frequently in our following algorithm. More specifically, for given positive constants $\beta>0, r_{j} \geq \beta$ for $j=1,2, \ldots, N+1$, let

$$
M=\left(\begin{array}{ccccc}
r_{1} & 0 & \cdots & \cdots & 0 \\
\beta & r_{2} & \ddots & \ddots & 0 \\
\vdots & \ddots & \ddots & \ddots & \vdots \\
-\beta & \cdots & -\beta & r_{N+1} & 0 \\
0 & 0 & \cdots & 0 & \frac{1}{\beta}
\end{array}\right) .
$$

The matrix $M$ defined by (13) is a nonsingular lower triangular matrix. Moreover, let

$$
H=\operatorname{diag}\left(r_{1}, r_{2}, \ldots, r_{N+1}, \frac{1}{\beta}\right) .
$$

Clearly, $H$ defined in (14) is a positive definite diagonal matrix. 
With the definitions depicted by (13) and (14), it is easy that we have the result below:

$$
H^{-1} M^{T}=\left(\begin{array}{cccccc}
1 & \frac{\beta}{r_{1}} & \frac{\beta}{r_{1}} & \cdots & -\frac{\beta}{r_{1}} & 0 \\
0 & 1 & \frac{\beta}{r_{2}} & \ddots & -\frac{\beta}{r_{2}} & 0 \\
\vdots & \ddots & \ddots & \ddots & \ddots & \vdots \\
0 & \ddots & \ddots & 1 & -\frac{\beta}{r_{N}} & 0 \\
0 & \cdots & 0 & 0 & 1 & 0 \\
0 & \cdots & 0 & 0 & 0 & 1
\end{array}\right) .
$$

Herein, formula (15) is an upper-triangular block matrix with diagonal components being identity equal to one. Accordingly, we present the algorithmic framework to solve (4).

Distributed Optimization Algorithm Based on ADMM-GBS. Each subscriber sends $x_{i}^{t}(k)$ to the energy provider, combined with $G_{k}^{t}$ and $\lambda_{k}^{t}$; the energy provider then gets the initial iteration $\zeta_{k}^{t}=\left(x_{1}^{t}(k), x_{2}^{t}(k), \ldots, x_{N}^{t}(k), G_{k}^{t}, \lambda_{k}^{t}\right)$, and the new iteration $\zeta_{k}^{t+1}=\left(x_{1}^{t+1}(k), x_{2}^{t+1}(k), \ldots, x_{N}^{t+1}(k), G_{k}^{t+1}, \lambda_{k}^{t+1}\right)$ is generated as follows.

Step 1. Obtain $\widetilde{\zeta}_{k}^{t}=\left(\widetilde{x}_{1}^{t}(k), \widetilde{x}_{2}^{t}(k), \ldots, \widetilde{x}_{N}^{t}(k), \widetilde{G}_{k}^{t}, \tilde{\lambda}_{k}^{t}\right)$ in the forward (alternating) order by the following ADMM procedure:

$$
\begin{aligned}
& \widetilde{x}_{i}^{t}(k)=\arg \min \left\{-\widehat{U}_{i}\left(x_{i}(k)\right)\right. \\
& +\frac{r_{i}}{2}\left\|x_{i}(k)-\left(x_{i}^{t}(k)+\frac{1}{r_{i}} q_{i}^{k}\right)\right\|^{2} \mid x_{i}(k) \\
& \left.\in\left[m_{i}^{k}, M_{i}^{k}\right]\right\}, \\
& \widetilde{G}_{k}^{t}=\arg \min \left\{C_{k}\left(G_{k}\right)\right. \\
& +\frac{r_{N+1}}{2}\left\|G_{k}-\left(G_{k}^{t}-\frac{1}{r_{N+1}} q_{G_{k}}\right)\right\|^{2} \mid G_{k} \\
& \left.\quad \in\left[G_{k}^{\min }, G_{k}^{\max }\right]\right\}, \\
& \widetilde{\lambda}_{k}^{t}=\lambda_{k}^{t}-\beta\left(\widetilde{G}_{k}^{t}-\sum_{j=1}^{N} \widetilde{x}_{j}^{t}(k)\right),
\end{aligned}
$$

for $i=1,2, \ldots, N$,

$$
\begin{aligned}
& q_{i}^{k}=\lambda_{k}^{t}-\beta\left(\sum_{j=1}^{i-1} \tilde{x}_{j}^{t}(k)+G_{k}^{t}-\sum_{j=i}^{N} x_{j}^{t}(k)\right), \\
& q_{G_{k}}=\lambda_{k}^{t}-\beta\left(G_{k}^{t}-\sum_{j=1}^{N} \tilde{x}_{j}^{t}(k)\right) .
\end{aligned}
$$

Step 2. Let $v \in(0,1)$, amend the output $\widetilde{\zeta}_{k}^{t}$ by the following Gaussian back substitution procedure, and generate the new iteration $\zeta_{k}^{t+1}$ :

$$
H^{-1} M^{T}\left(\zeta_{k}^{t+1}-\zeta_{k}^{t}\right)=v\left(\widetilde{\zeta}_{k}^{t}-\zeta_{k}^{t}\right)
$$

Step 3. Stop criteria

$$
\begin{gathered}
\left|\lambda_{k}^{t+1}-\lambda_{k}^{t}\right|<\varepsilon_{1}, \\
\left|\zeta_{k}^{t+1}-\zeta_{k}^{t}\right|<\varepsilon_{2} .
\end{gathered}
$$

The convergence of the proposed distributed optimization algorithm based on ADMM-GBS can be guaranteed by the following theorem. The next theorem will use a symmetric and positive semidefinite matrix defined as

$$
Q=\left(\begin{array}{cccccc}
\beta & \beta & \cdots & \beta & -\beta & 1 \\
\vdots & \vdots & \ddots & \vdots & \vdots & \vdots \\
\beta & \beta & \cdots & \beta & -\beta & 1 \\
-\beta & -\beta & \cdots & -\beta & \beta & -1 \\
1 & 1 & \cdots & 1 & -1 & \frac{1}{\beta}
\end{array}\right) .
$$

Theorem 2. Let $\widetilde{\zeta}_{k}^{t}=\left(\widetilde{x}_{1}^{t}(k), \widetilde{x}_{2}^{t}(k), \ldots, \widetilde{x}_{N}^{t}(k), \widetilde{G}_{k}^{t}, \widetilde{\lambda}_{k}^{t}\right)$ be generated by the ADMM procedure in formulae (16), (17), and (18) from the given vector $\zeta_{k}^{t}=\left(x_{1}^{t}(k), x_{2}^{t}(k), \ldots, x_{N}^{t}(k), G_{k}^{t}, \lambda_{k}^{t}\right)$, $\forall k \in \mathbb{K}$, and $\left\{\zeta_{k}^{t}\right\}$ be the sequence generated by the proposed algorithm. Denote $\|x\|_{Q}:=\sqrt{x^{T} Q x} ; \forall \zeta_{k}^{*} \in R^{N+2}$, one has

$$
\begin{aligned}
& \left(\zeta_{k}^{t}-\zeta_{k}^{*}\right)^{T} M\left(\zeta_{k}^{t}-\widetilde{\zeta}_{k}^{t}\right) \\
& \geq \frac{1}{2}\left\|\zeta_{k}^{t}-\widetilde{\zeta}_{k}^{t}\right\|_{H}^{2}+\frac{1}{2}\left\|\zeta_{k}^{t}-\widetilde{\zeta}_{k}^{t}\right\|_{Q}^{2}, \\
& \left\|\zeta_{k}^{t+1}-\zeta_{k}^{*}\right\|_{G}^{2} \\
& \leq\left\|\zeta_{k}^{t}-\zeta_{k}^{*}\right\|_{G}^{2}-c_{0}\left(\left\|\zeta_{k}^{t}-\widetilde{\zeta}_{k}^{t}\right\|_{H}^{2}+\left\|\zeta_{k}^{t}-\widetilde{\zeta}_{k}^{t}\right\|_{Q}^{2}\right),
\end{aligned}
$$

where $G=M H^{-1} M^{T}$ and $c_{0}=v(1-v)>0$. Furthermore, the sequence $\left\{\zeta_{k}^{t}\right\}$ is monotone and satisfies $\lim _{t \rightarrow \infty}\left\|\zeta_{k}^{t}-\widetilde{\zeta}_{k}^{t}\right\|=0$. In addition, $\left\{\zeta_{k}^{t}\right\}$ converges to the solution of problem (4).

Proof. Formula (21) can be rewritten as $\zeta_{k}^{t+1}=\zeta_{k}^{t}-$ $M^{-T} H v\left(\zeta_{k}^{t}-\widetilde{\zeta}_{k}^{t}\right)$. Cited from the literature by He and Yuan [26], conclusions of (24) and (25) can be obtained. Following (24), we have

$$
\begin{aligned}
& \left\langle M H^{-1} M^{T}\left(\zeta_{k}^{t}-\zeta_{k}^{*}\right), M^{-T} H\left(\widetilde{\zeta}_{k}^{t}-\zeta_{k}^{*}\right)\right\rangle \\
& \quad \leq-\frac{1}{2}\left\|\zeta_{k}^{t}-\widetilde{\zeta}_{k}^{t}\right\|_{H+Q}^{2}
\end{aligned}
$$

This ensures $-M^{-T} H v\left(\zeta_{k}^{t}-\widetilde{\zeta}_{k}^{t}\right)$ is a descent direction of function $(1 / 2)\left\|\zeta-\zeta_{k}^{*}\right\|_{G}^{2}$ at point $\zeta=\zeta_{k}^{t}$ while $\widetilde{\zeta}_{k}^{t} \neq \zeta_{k}^{t}$, where $\widetilde{\zeta}_{k}^{t}$ is generated by a procedure in formulae (16), (17), 
Step 1. When $k \in \mathbb{K}$, for each user $i \in \mathbb{N}$, initializes $x_{i}^{t}(k) \in\left[m_{i}^{k}, M_{i}^{k}\right]$, and energy provider announces $\lambda_{k}^{t} \geq 0, G_{k}^{t} \geq 0$ to each user.

Step 2. In time slot $k$, each user does the following:

(1) Updates the value of $q_{i}^{k}$ by (19);

(2) Solves local problem (16) to obtain $\tilde{x}_{i}^{t}(k)$;

(3) Broadcasts $\widetilde{x}_{i}^{t}(k)$ to each user and energy provider.

Step 3. In time slot $k$, energy provider does the following:

(1) Updates $q_{G_{k}}$ via (20);

(2) Receives $\widetilde{x}_{i}^{t}(k)$ from all the users, solves problem (17) to get $\widetilde{G}_{k}^{t}$, updates the value of $\widetilde{\lambda}_{k}^{t}$ by (18);

(3) Broadcasts $\widetilde{G}_{k}^{t}$ and $\widetilde{\lambda}_{k}^{t}$ to the users.

Step 4. Energy provider corrects $\widetilde{\zeta}_{k}^{t}$ via (21) to get $\zeta_{k}^{t}$.

Step 5. If stop criteria (22) are satisfied, algorithm stops. Otherwise, repeats Steps 2 to 5.

Algorithm 1: Distributed optimization algorithm based on ADMM-GBS.

and (18), $\zeta_{k}^{*} \in R^{N+2}$ and $G=M H^{-1} M^{T}$. The conclusion in (25) shows that sequence $\left\{\zeta_{k}^{t}\right\}$ generated by this algorithm is contractive with respect to $R^{N+2}$, which means $\lim _{t \rightarrow \infty} \| \zeta_{k}^{t}-$ $\widetilde{\zeta}_{k}^{t} \|=0$. Furthermore, these conditions are adequate to ensure convergence of the distributed optimization algorithm based on ADMM-GBS [26].

Finally, a summarization of the distributed optimization algorithm based on ADMM-GBS is depicted in Algorithm 1.

\section{Numerical Evaluation}

In this section, we illustrate the performance of the distributed optimization algorithm based on ADMM-GBS by providing several results of the numerical simulations. In order to give the range of parameters, realistic hourly load profiles have been considered in this paper. Sourcing the residential load data published by Open EI, we obtained/developed samples of hourly load profiles of residential homes [27]. Figure 1 shows a sample in the load profiles. It reflects a user's power consumption at each slot (24 hours, the horizontal axis) of a year (365 days, the vertical axis). The range of the user's hourly power demand is $x_{i}(k) \epsilon$ $[0.5,4.5]$. The lower power consumption of the residential user is identified as blue while the higher one is identified as red. The peak demand moments for the user can be recognized easily.

Considering a smart grid system in a small area with $N=$ 5 , that is, 5 users, we show numerical simulation results within a 24-hour time pattern as an evaluation of daily operations. The utility function of the user is chosen as follows with $\omega_{i}(k) \in[1,3.5]:$

$$
\begin{aligned}
& U_{i}\left(x_{i}(k)\right) \\
& \quad= \begin{cases}\omega_{i}(k) x_{i}(k)-\frac{1}{8} x_{i}(k)^{2}, & 0 \leq x_{i}(k) \leq 4 \omega_{i}(k), \\
2 \omega_{i}(k)^{2}, & x_{i}(k) \geq 4 \omega_{i}(k) .\end{cases}
\end{aligned}
$$

Since the utility function is quadratic and the feasible sets are intervals, the explicit solutions for $x_{i}{ }^{*}(k), G_{k}^{*}$, and $\lambda_{k}^{*}$ are given [10], and $\lambda_{k}^{*}=\left(2 a_{k} \sum_{i=1}^{N} \omega_{i}(k)+\alpha_{i} b_{k}\right) /\left(2 a_{k} N+\alpha_{i}\right)$, we

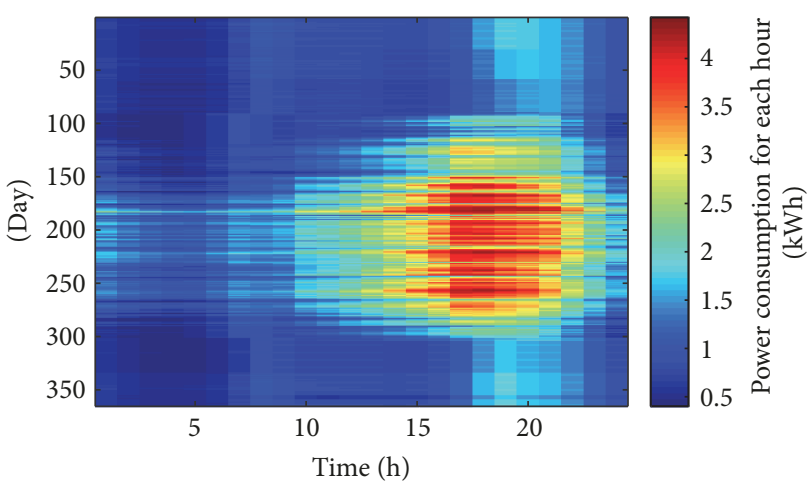

FIGURE 1: Resident user's electricity consumption at each slot of a year.

obtain that $\lambda_{k}^{\min } \leq \lambda_{k}^{*} \leq \lambda_{k}^{\max }, \lambda_{k}^{\min }=\left(2 a_{k} N \cdot \min \left(\omega_{i}(k)\right)+\right.$ $\left.\alpha_{i} b_{k}\right) /\left(2 a_{k} N+\alpha_{i}\right)$, and $\lambda_{k}^{\max }=\left(2 a_{k} N \cdot \max \left(\omega_{i}(k)\right)+\right.$ $\left.\alpha_{i} b_{k}\right) /\left(2 a_{k} N+\alpha_{i}\right)$, when $N \rightarrow \infty, \lambda_{k}^{\min } \rightarrow \min \left(\omega_{i}(k)\right)$, and $\lambda_{k}^{\max } \rightarrow \max \left(\omega_{i}(k)\right)$. Therefore, we may choose $\lambda_{k} \in[1,3.5]$, which is identical to $\omega_{i}(k)$.

From the actual application, there is no doubt that the objective function is greater than zero. This means $\sum_{i=1}^{N} U_{i}\left(x_{i}(k)\right)-C_{k}\left(G_{k}\right) \geq 0$. This implies that the parameters involved in the model need to satisfy the inequality $\sum_{i=1}^{N}\left(\omega_{i}(k) m_{i}^{k}-\left(\alpha_{i} / 2\right) m_{i}^{k}\right)-c+b_{k}^{2} / 4 a_{k} \geq a_{k}\left(\sum_{i=1}^{N} m_{i}^{k}+b_{k} / 2 a_{k}\right)^{2}$.

Based on this hypothesis, we assume that the user's energy demand $x_{i}(k)$ is selected from the set of $[0.5,4.5]$ for all $i \in \mathbb{N}$. The cost parameters are $a_{k}=0.05$ and $b_{k}=c_{k}=0, \forall k \in \mathbb{K}$. Two situations are analyzed, calculated, and discussed per required accuracy selection $\varepsilon_{1}=\varepsilon_{2}=\varepsilon=0.03$ or $\varepsilon_{1}=$ $\varepsilon_{2}=\varepsilon=0.001$. Notice that, for each time slot $k$, we have a terminating condition for the inner loop; that is, $\left|\lambda_{k}^{t+1}-\lambda_{k}^{t}\right|<$ $\varepsilon(\forall \varepsilon>0)$, where $\varepsilon$ is a positive real number small enough to indicate the convergence of $\lambda_{k}^{t}$.

As shown in Figures 2 and $3, \varepsilon$ has a great influence on the iteration times for the subgradient algorithm. It presents a very slow convergent speed and even nonconvergence during the iteration process. When $\varepsilon=0.001$, the reference price (green line) converges at the 126th iteration. When $\varepsilon=0.03$, 


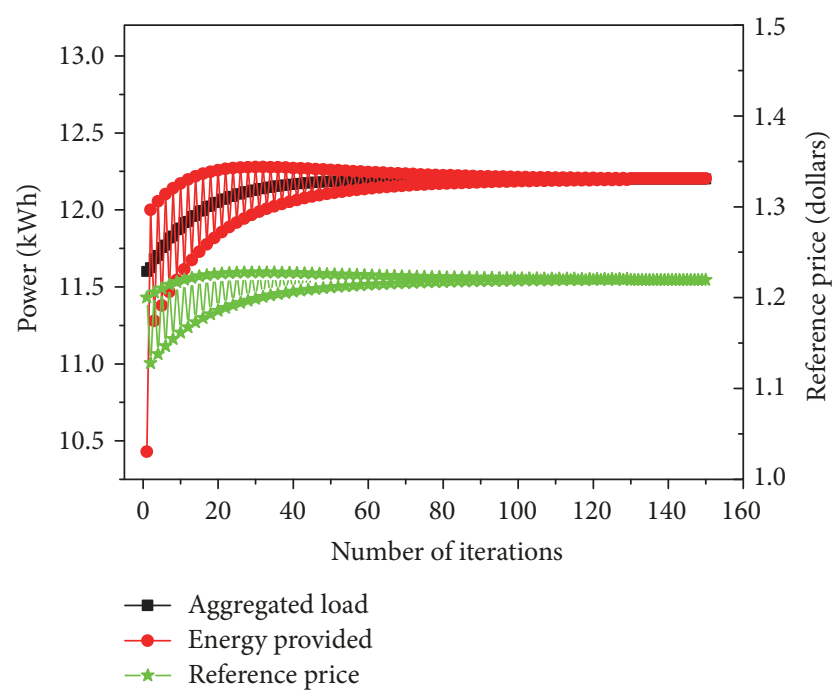

Figure 2: Convergence of the subgradient algorithm when $\varepsilon=$ 0.001 .

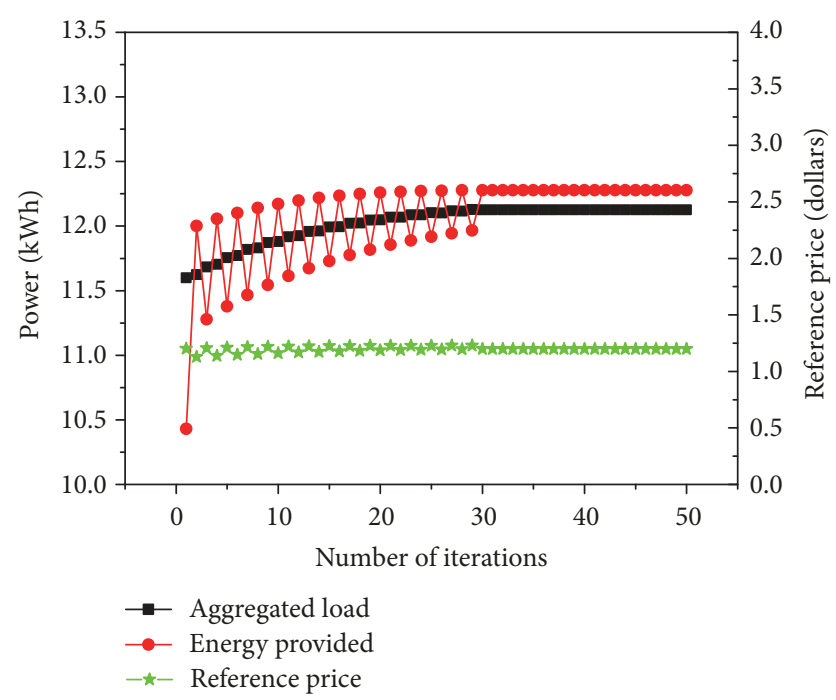

FIGURE 3: Convergence of the subgradient algorithm when $\varepsilon=0.03$.

the system reaches convergence at 30 times. A smaller $\varepsilon$ value produces a more precise optimal solution, but its iteration process costs more steps and takes much more time on computation than a larger $\varepsilon$ value does. The aggregated load (black line) fluctuates with reference price of electricity. There is a certain deviation between optimal electricity production (red line) and aggregated load while $\varepsilon=0.03$.

The other factor that affects the convergence of the iteration is step size $\beta$. The subgradient algorithm suffers from slow convergence or even nonconvergence and is numerically sensitive to $\beta[1]$. Furthermore, this research found that a step size is suitable for one time slot but may be inappropriate for another time slot through the simulation. One of the advantages of the distributed optimization algorithm based

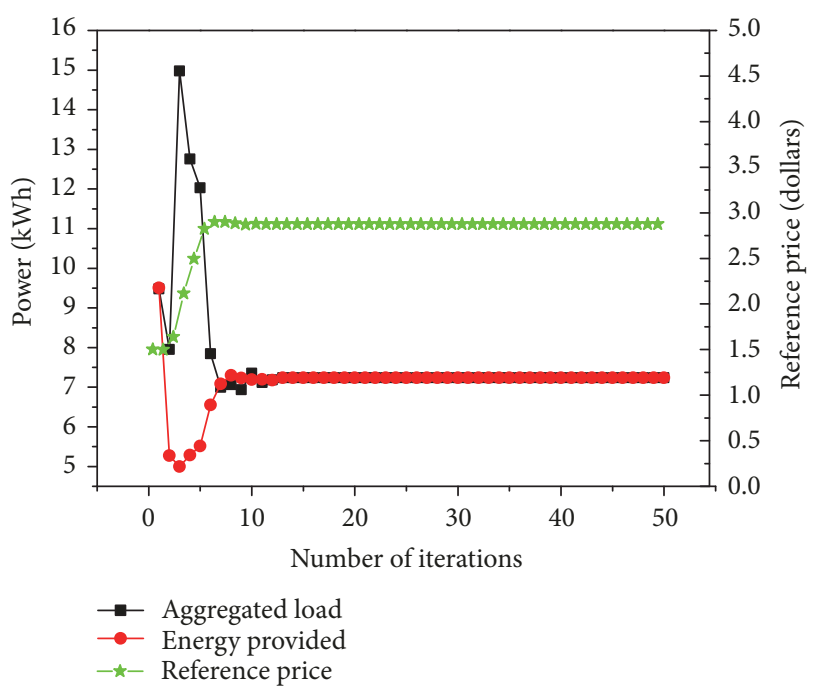

FIGURE 4: Convergence of the distributed optimization algorithm based on ADMM-GBS.

on ADMM-GBS is that it can overcome efficiently the demerits of the subgradient algorithm described above. We choose step size $\beta$ to be randomly selected in $[0.01,0.1]$, and $r_{j}=\beta+\delta$, where $\delta$ is randomly selected from the set of $[0,0.1]$. The deviation generated by $\beta$ could be eliminated during the process of GBS. It guarantees the convergence of the algorithm.

As shown in Figure 4, our numerical experiment verifies that the distributed optimization algorithm based on ADMM-GBS is time-saving and has a rapid convergence rate while meeting the required accuracy $\varepsilon=0.001$. At the same time, it verifies its low dependence on initial value. At the beginning of the iteration, the price of electricity (green line) is very low. Thus, the high aggregated load (black line) results from the cheap price while the optimized generation of electricity (red line) is low. With the signal of increasing price, the difference between load and generation becomes narrower and narrower and finally disappears after meeting the termination criteria. This algorithm reflects the varying process of iteration more clearly. Through clear illustration of the dynamic iteration process shown in Figure 4, the proposed algorithm presents a faster convergence rate than the subgradient algorithm shown in Figures 2 and 3. Fast convergence is critically important for a large-scale system with a large population of users in customer domain, because its requested speed on information exchange between the energy provider and individual users is much higher than in a small-scale system.

Figure 5 shows the performance of optimal electricity generation (black line), user's power consumption (red line), and referential RTP (green line) in a typical day. Based on Figure 5, the following conclusions could be reached. On the one hand, RTP is closely related to the aggregated load of users at each slot. Through the implementation of RTP, the price signal may guide customers and motivate them to consume more energy in off-peak periods. It not only reduces peak load for the energy provider, thus achieving its primary 


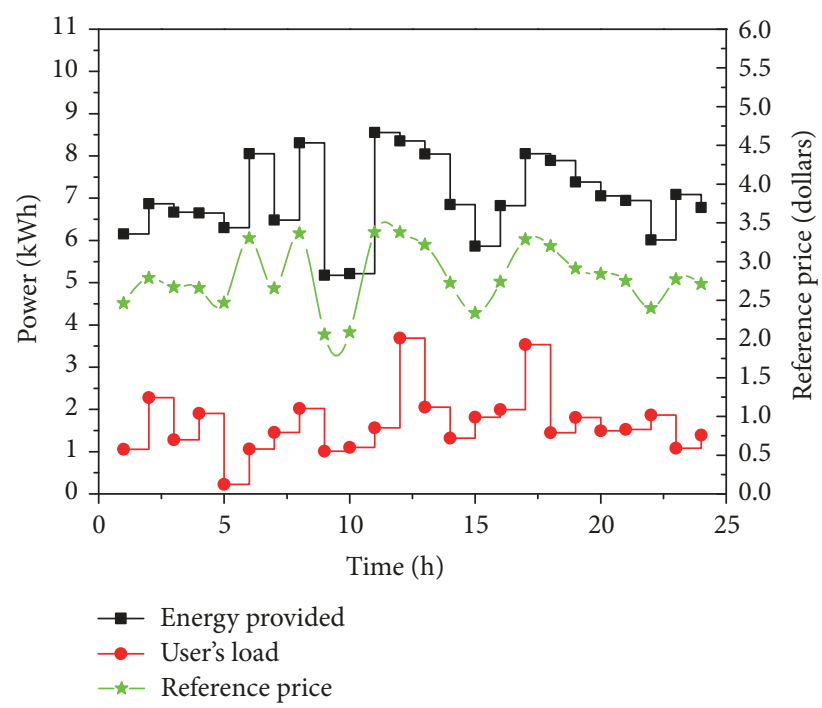

Figure 5: Optimal solutions of the distributed optimization algorithm based on ADMM-GBS.

goal of cutting peak and fulfilling the ideal demand of power load, but it reduces the electricity bill for the users also. Fundamentally, it ends up benefiting the social community by achieving the target on energy conservation and emission reduction. On the other hand, the RTP depicted in the simulation presents three peak demands per day. It verifies the rationality of RTP optimization model and the validity of algorithm this study proposed.

\section{Conclusion}

This study has proposed the distributed optimization algorithm based on ADMM-GBS for solving the RTP model in a smart grid system. To enhance the robustness of the subgradient algorithm and ensure yielding convergence of the algorithm, the augmented Lagrangian function has been utilized in this research. This study has analyzed the existence and uniqueness of the Lagrangian multiplier and concluded that the local optimal solution and related Lagrangian multiplier are all unique in the local region. The distributed optimization algorithm based on ADMMGBS utilized in this research can not only make abundant use of the separability of variables, but also improve the convergence speed of the iteration. In addition, we have given the theoretical proof of the convergence of the proposed algorithm. Most importantly, the proposed algorithm inherits the advantage of the distributed method which protects individual users' private information. The interdependent relationship between variables has been discussed in depth during the numerical simulation in the study. Furthermore, the numerical simulation results (a) verify that the RTP method can guide customers' consumption behavior and achieve our primary goal on cutting peak, which benefits social community's target on energy conservation and emission reduction, and (b) verify the rationality and validity of the proposed algorithm.

\section{Conflicts of Interest}

The authors declare that there are no conflicts of interest regarding the publication of this paper.

\section{Acknowledgments}

This work was supported by the National Natural Science Foundation of China (no. 11171221), Natural Science Foundation of Shanghai (14ZR1429200), and Innovation Program of Shanghai Municipal Education Commission (15ZZ074).

\section{References}

[1] W. Zhang, J. Li, G. Chen, Z. Y. Dong, and K. P. Wong, "A comprehensive model with fast solver for optimal energy scheduling in RTP environment," IEEE Transactions on Smart Grid, vol. 8, no. 5, pp. 2314-2323, 2017.

[2] Y. Aiwei, X. Congwei, and J. Liwei, "A joint scheduling optimization model for wind power and energy storage systems considering carbon emissions trading and demand response," Mathematical Problems in Engineering, vol. 2016, Article ID 4070251, 10 pages, 2016.

[3] Z.-F. Tan, Y.-H. Song, H.-J. Zhang, Q.-S. Shi, and J. Xu, "Joint optimization model of generation side and user side based on energy-saving policy," International Journal of Electrical Power \& Energy Systems, vol. 57, pp. 135-140, 2014.

[4] M. Mahdavian and N. Wattanapongsakorn, "Optimizing greenhouse lighting for advanced agriculture based on real time electricity market price," Mathematical Problems in Engineering, vol. 2017, Article ID 6862038, 11 pages, 2017.

[5] M. C. Caramanis, R. E. Bohn, and F. C. Schweppe, "Optimal spot pricing: practice and theory," IEEE Transactions on Power Apparatus and Systems, vol. 101, no. 9, pp. 3234-3245, 1982.

[6] P. Samadi, H. Mohsenian-Rad, R. Schober, V. Wong, and J. Jatskevich, "Optimal real-time pricing algorithm based on utility maximization for smart grid," in Proceedings of the 1st IEEE International Conference on Smart Grid Communications (SmartGridComm '10), pp. 415-420, Gaithersburg, Md, USA, October 2010.

[7] P. Samadi, H. Mohsenian-Rad, R. Schober, and V. W. S. Wong, "Advanced demand side management for the future smart grid using mechanism design," IEEE Transactions on Smart Grid, vol. 3, no. 3, pp. 1170-1180, 2012.

[8] A. Mohsenian-Rad, V. Wong, J. Jatskevich, R. Schober, and A. LeonGarcia, "Autonomous demand-side management based on game-theoretic energy consumption scheduling for the future smart grid," IEEE Transactions on Smart Grid, vol. 1, no. 3, pp. 320-331, 2010.

[9] X. Song and J. Qu, "An improved real-time pricing algorithm based on utility maximization for smart grid," in Proceedings of the 2014 11th World Congress on Intelligent Control and Automation, WCICA 2014, pp. 2509-2513, China, July 2014.

[10] P. Tarasak, "Optimal real-time pricing under load uncertainty based on utility maximization for smart grid," in Proceedings of the IEEE 2nd International Conference on Smart Grid Communications (SmartGridComm '11), pp. 321-326, October 2011.

[11] Y. Wang, S. Mao, and R. M. Nelms, "Distributed online algorithm for optimal real-time energy distribution in the smart grid," IEEE Internet of Things Journal, vol. 1, no. 1, pp. 70-80, 2014. 
[12] G. Asadi, M. Gitizadeh, and A. Roosta, "Welfare maximization under real-time pricing in smart grid using PSO algorithm," in Proceedings of the 2013 21st Iranian Conference on Electrical Engineering, ICEE 2013, Iran, May 2013.

[13] S.-C. Tsai, Y.-H. Tseng, and T.-H. Chang, "Communicationefficient distributed demand response: a randomized ADMM approach," IEEE Transactions on Smart Grid, vol. 8, no. 3, pp. 1085-1095, 2017.

[14] Z. Tan, P. Yang, and A. Nehorai, "An optimal and distributed demand response strategy with electric vehicles in the smart grid," IEEE Transactions on Smart Grid, vol. 5, no. 2, pp. 861869, 2014.

[15] C. Feng, Z. Li, M. Shahidehpour, F. Wen, W. Liu, and X. Wang, "Decentralized Short-term Voltage Control in Active Power Distribution Systems," IEEE Transactions on Smart Grid, vol. 99, pp. 1-10, 2017.

[16] Y. Wang, L. Wu, and S. Wang, "A fully-decentralized consensusbased ADMM approach for DC-OPF with demand response," IEEE Transactions on Smart Grid, vol. 8, no. 6, pp. 2637-2647, 2017.

[17] D. H. Nguyen, T. Narikiyo, and M. Kawanishi, "Optimal Demand Response and Real-time Pricing by a Sequential Distributed Consensus-based ADMM Approach," IEEE Transactions on Smart Grid, vol. 99, pp. 1-11, 2017.

[18] U. S. Department of Commerce, "Guidelines for smart grid cyber security," Privacy and the Smart Grid, pp. 1-8, 2010.

[19] S. Boyd, N. Parikh, E. Chu, B. Peleato, and J. Eckstein, "Distributed optimization and statistical learning via the alternating direction method of multipliers," Foundations and Trends in Machine Learning, vol. 3, no. 1, pp. 1-122, 2010.

[20] M. R. Hestenes, "Multiplier and gradient methods," Journal of Optimization Theory and Applications, vol. 4, no. 5, pp. 303-320, 1969.

[21] M. J. D. Powell, "A method for nonlinear constraints in minimization problems," in Optimization. A Journal of Mathematical Programming and Operations Research, R. Fletcher, Ed., pp. 283-298, Academic Press, New York, NY, USA, 1969.

[22] S. Boyd and L. Vandenberghe, Convex Optimization, Cambridge University Press, 2004.

[23] M. Fukushima, Nonlinear Optimization, Science Press, China, 2011.

[24] L. Li, X. Wang, and G. Wang, "Alternating direction method of multipliers for separable convex optimization of real functions in complex variables," Mathematical Problems in Engineering, vol. 2015, Article ID 104531, 14 pages, 2015.

[25] S. Setzer, G. Steidl, and T. Teuber, "Deblurring poissonian images by split Bregman techniques," Journal of Visual Communication and Image Representation, vol. 21, no. 3, pp. 193-199, 2010.

[26] B. He and X. Yuan, "Linearized alternating direction method of multipliers with Gaussian back substitution for separable convex programming," Numerical Algebra, Control and Optimization, vol. 3, no. 2, pp. 247-260, 2013.

[27] Open EI, "Residential Load Data," http://en.openei.org/wiki/ Main_Page. 


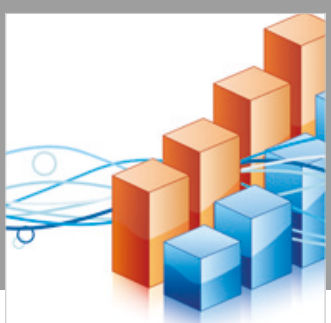

Advances in

Operations Research

\section{-n-m}
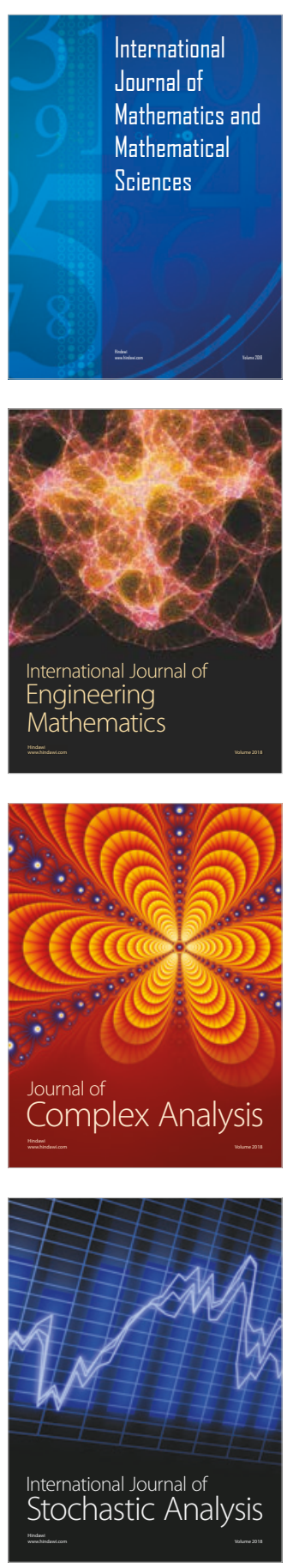
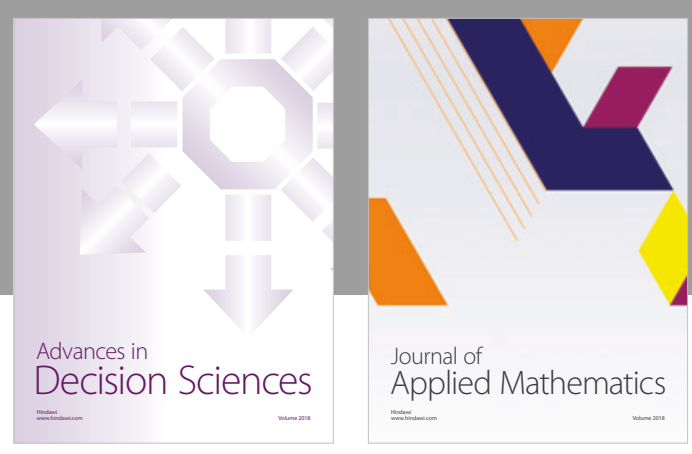

Journal of

Applied Mathematics
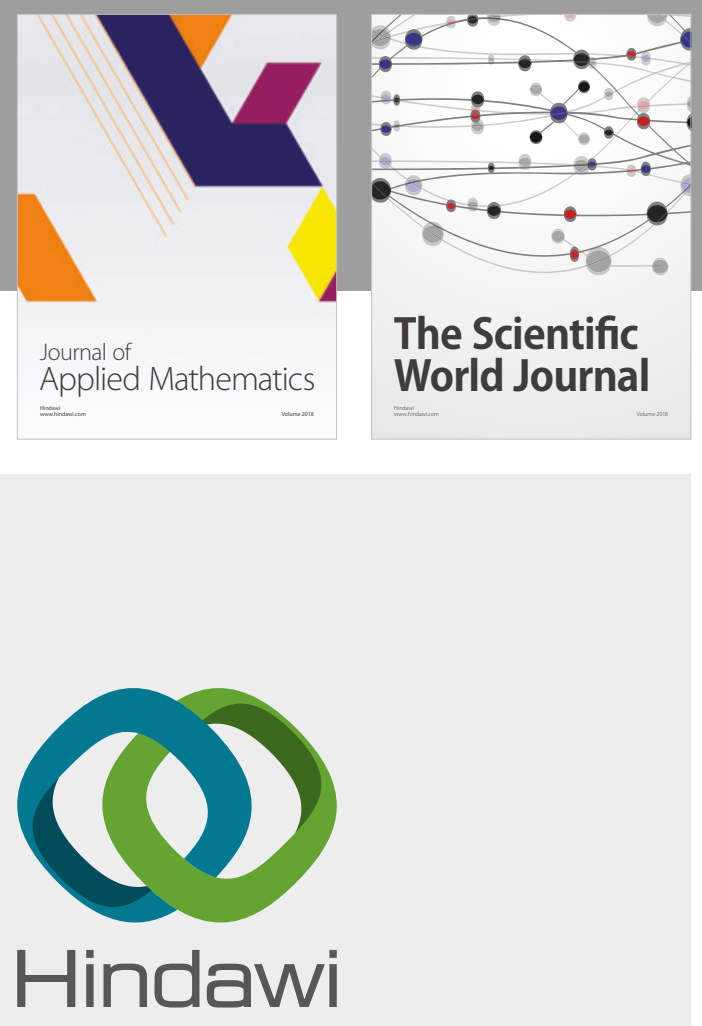

Submit your manuscripts at

www.hindawi.com

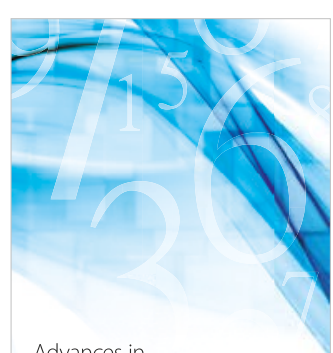

Advances in
Numerical Analysis
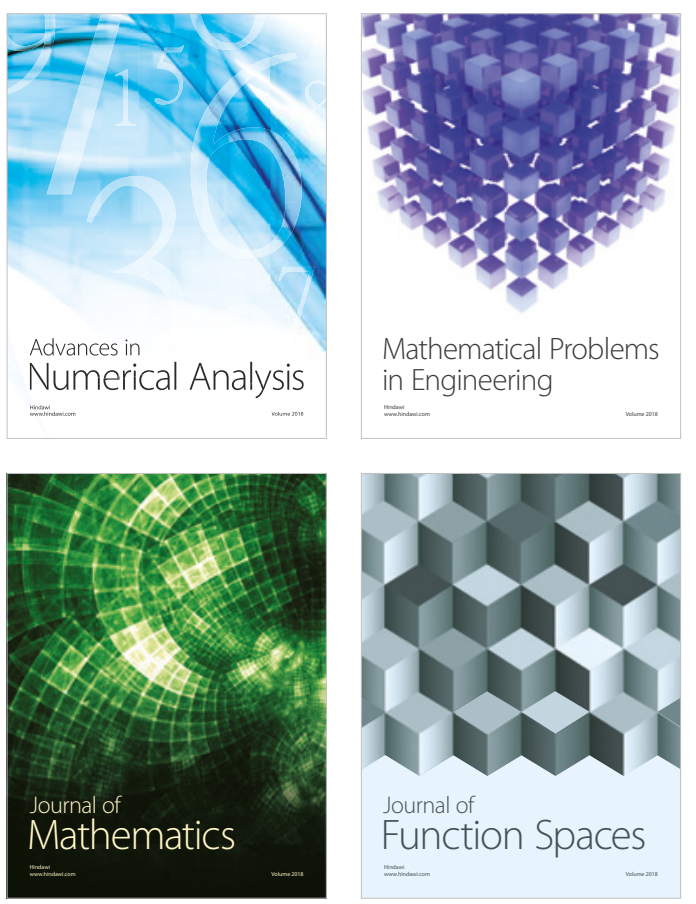

Mathematical Problems in Engineering

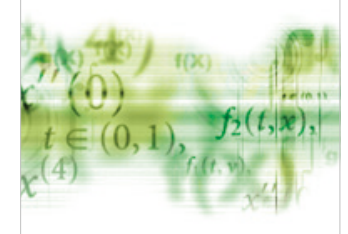

International Journal of

Differential Equations

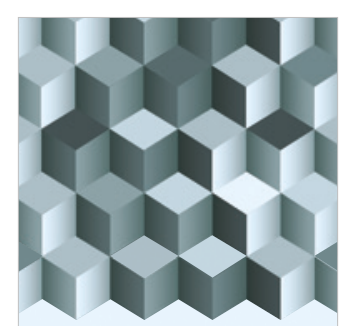

Journal of

Function Spaces

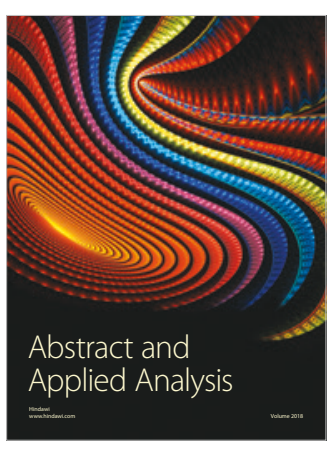

The Scientific

World Journal

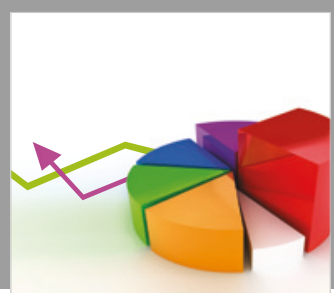

Journal of

Probability and Statistics
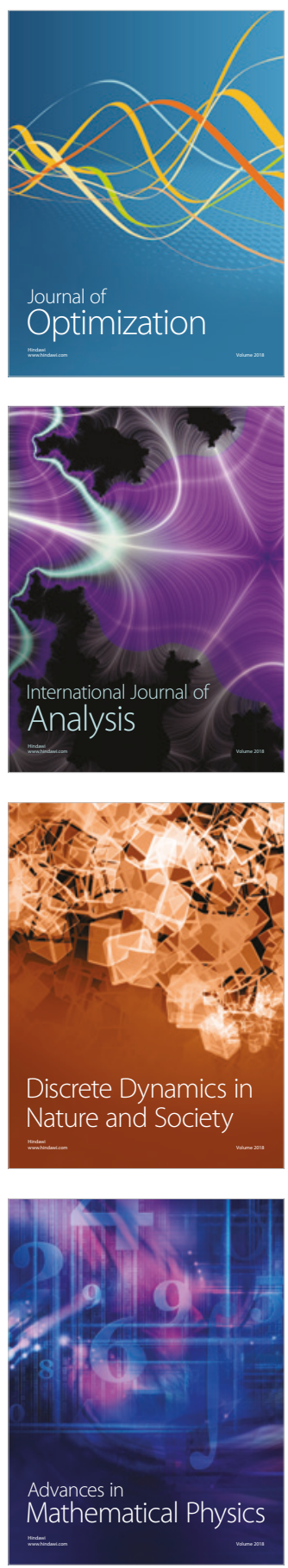